\title{
Research Paper: The Relationship Between Multiple Dimensions of Schizotypy and Obsessive-Compulsive Symptoms: The Mediating Role of Inferential Confusion
}

\author{
Akram Ghorbali $^{1^{*}}$ (1D) \\ 1. Department of Psychology, Faculty of Psychology and Educational Sciences, South Tehran Branch, Islamic Azad University, Tehran, Iran.
}

\begin{tabular}{|c|c|}
\hline $\begin{array}{l}\text { Use your device to scan } \\
\text { and read the article online }\end{array}$ & ditation Ghorbali, A. (2021). The Relationship Between Multiple Dimensions of Schizotypy and Obsessive-Compulsive \\
\hline a:yetra & $\begin{array}{l}\text { Symptoms: The Mediating Role of Inferential Confusion. Journal of Practice in Clinical Psychology, 9(4), 313-320. https://doi. } \\
\text { org/10.32598/jpcp.9.4.786.1 }\end{array}$ \\
\hline 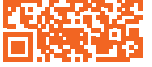 & dol'https://doi.org/10.32598/jpcp.9.4.786.1 \\
\hline
\end{tabular}

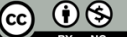

Article info:

Received: 15 Aug 2021

Accepted: 10 Sep 2021

Available Online: 01 Oct 2021

Keywords:

Schizotypal personality disorder, Obsessive behavior, Compulsive behavior, Confusion

\section{ABSTRACT}

Objective: The relationship between obsessive-compulsive and schizotypal symptoms has been repeatedly mentioned in various studies, but the exact pattern of this relationship is still unclear. Recently, the Inference-Based Approach (IBA) tries to explain this relationship through Inferential Confusion (IC). This study aimed to determine the mediating role of inferential confusion in the relationship between different dimensions of schizotypy and Obsessive-Compulsive Symptoms (OCS).

Methods: The study sample consisted of 341 (158 men and 183 women) university students of Shahed University, Tehran, Iran. Participants were randomly selected by cluster sampling method and completed Schizotypal Personality Questionnaire-Brief (SPQ-B), Inferential Confusion Questionnaire-Expanded Version (ICQ-EV), and Obsessive-Compulsive InventoryRevised (OCI-R). The proposed model was examined by Structural Equation Modeling (SEM).

Results: Model fit indices $\left(\mathrm{GFI}=0.91, \mathrm{RMSEA}=0.04, \mathrm{X}^{2} / \mathrm{df}=1.54\right)$ indicated good fit. Results showed that among the three dimensions of schizotypy, only the direct effect of positive schizotypy on obsessive-compulsive symptoms $(\beta=0.60, \mathrm{P}<0.001)$ and inferential confusion ( $\beta=0.64, \mathrm{P}<0.001)$ were statistically significant. Also, Bootstrap method showed that only the indirect effect of positive schizotypy on OCS was statistically significant $[\mathrm{CI}(95 \%)=0.01-0.25]$.

Conclusion: Consistent with previous findings, it seems that the relationship between OCS and schizotypy is meaningful along the positive dimension of schizotypy and inferential confusion can explain this relationship to some extent.

\section{* Corresponding Author:}

Akram Ghorbali, PhD.

Address: Department of Psychology, Faculty of Psychology and Educational Sciences, South Tehran Branch, Islamic Azad University, Tehran, Iran.

Tel: +98 (991) 2080045

E-mail:s.ghorbali@yahoo.com 


\section{Highlights}

- In the relationship between Oobsessive-Compulsive Symptoms (OCS) and schizotypy, attention should be paid to the multi-dimensional nature of schizotypy.

- Inferential Confusion (IC) mediates the relationship between OCS and positive schizotypy.

- Only the direct effect of positive schizotypy on OCS and IC was statistically significant.

\section{Plain Language Summary}

Despite the numerous pieces of evidence for the relationship between Obsessive-Compulsive Symptoms (OCS) and schizotypal personality traits, there is no coherent conceptualization about the nature of the relationship between these two variables. Research has confirmed the comorbidity and structural similarity between these two groups of symptoms. This, in turn, provides some basic implications for the treatment of patients with symptoms of both disorders. In addition, research evidence suggests that schizotypy has a multi-dimensional structure and it is recommended to pay attention to its various dimensions in relation to OCS. Based on the Inference-Based Approach (IBA), Inferential Confusion (IC), as an imaginative process, plays a significant role in explaining the relationship between OCS and schizotypy. The findings of this study showed that only the direct and indirect (through IC) effects of the positive schizotypy on OCS were statistically significant and provided evidence to support the role of IC in the relationship between these two disorders.

\section{Introduction}

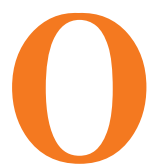

bsessive-Compulsive Disorder (OCD) is closely related to psychotic-like patterns of schizotypal personality (Tallis \& Shafran, 1997). The co-occurrence between Schizotypal Personality Disorder (SPD) and OCD in the different studies has been reported to vary from 5 to $50 \%$ (Perris et al., 2019). Also, the co-occurring rate between Obsessive-Compulsive Symptoms (OCS) and schizotypal traits in a student population was reported at $3.33 \%$ by Shan et al. (2020). In addition to the co-occurrence of the symptoms of these two disorders, some evidence suggests that there is a structural similarity between these two phenomena and they share a common psychological foundation (Mohammadzadeh, 2010). There are pieces of evidence that show OCD patients with and without schizotypal traits have differences in social and clinical characteristics. Earlier onset of the disorder, male gender, being unmarried and unemployed, having a history of specific phobia, and higher prevalence of counting compulsions are associated with an increased risk of schizotypy among OCD patients (Poyurovsky, 2013). A review of research literature shows that the relationship between schizotypal traits and OCS has extensively been investigated in clinical and non-clinical samples (Chmielewski \& Watson, 2008; Norman, Davies, Malla, Cortese, \& Nicholson, 1996; Raynal, Goutaudier, Nidetch, \& Chab- rol, 2016; Rossi \& Daneluzzo, 2002; Sobin et al., 2000; Weintraub, Brown \& Timpano, 2018), but the nature of this relationship is still not clear. In other words, investigating the relationship between OCD and SPD is not new, but identifying the underlying causes of these two disorders is a new and growing issue that helps increase basic knowledge about overlapping psychopathological features of them and has important implications for treating patients with symptoms of the two disorders.

In terms of the Inference-Based Approach (IBA; O’Connor \& Robillard, 1995), "Inferential Confusion' (IC), as a particular reasoning style, is one of the structural similarities between obsessive-compulsiveness and schizotypy. Based on this feature in the thinking process, people do not trust the information obtained from their senses when reasoning and invest in remote possibilities (Aardema, Emmelkamp, \& O’Connor, 2005). Based on this approach, some schizotypal features can be explained using the IC. This feature causes people to confuse reality with a possibility and then to feel and act on the basis of a series of internal narratives that are far from reality in here and now. As this style of reasoning intensifies, people become so far removed from the reality that they may experience some perceptual disturbances that characterize schizotypy (Aardema et al., 2005; Aardema, Kleijer, Trihey, O’Connor, \& Emmelkamp, 2006). Proponents of IBA believe that IC might have an underlying 
role in SPD and OCD and they suggest that both disorders may be belief disorders (O’Leary, 2015).

The relationship between inferential confusion, obsessive-compulsiveness, and schizotypy have been shown in some studies (e.g. Aardema et al., 2006; Aardema, \& Wu, 2011; O’Leary, 2015; Paradisis, Aardema, \& Wu, 2015), but these studies have considered the schizotypal personality as a one-dimensional, homogeneous construct. Since schizotypy has been identified as a three-dimensional construct, it is best to consider the different dimensions of it, including positive, negative, and disorganized. These three dimensions correspond to the cognitive-perceptual features (such as unusual perceptual experiences, ideas of reference, paranoid ideation, and magical thinking), interpersonal features (such as constricted affect, social anxiety, and lack of close friends), and disorganized features (odd behavior and speech), respectively (Raine \& Benishay, 1995). Clinical and non-clinical studies have shown that positive symptoms of schizotypy, such as magical thinking, suspiciousness, mistrust, and unusual beliefs are more associated with OCS (Chmielewski et al., 2005; Shan et al., 2020; Suhr, Spitznagel, Gunstad, 2006; Zhang et al., 2019). In addition, some factors associated with the poor prognosis and severity of OCD (such as the onset of the disorder at an early age, resistance to treatment, and having more comorbid disorders) have shown a strong relationship with positive schizotypal symptoms (Mohammadzadeh, Borjali, Sohrabi, Delavar, 2009; Moritz et al., 2004; Sobin et al., 2000).

The overlap in some features of psychopathology in these two distinct disorders and the significant comorbidity between them in the clinical and non-clinical populations suggests that they may share some common psychological foundations. Poyurovsky (2013) believes that examining the relationship between the symptoms of these two disorders in the general population regardless of the influence of intervening factors, such as the severity of symptoms and psychiatric treatments helps to increase our understanding of the nature of this relationship. Accordingly, this study intends to investigate the relationship between obsessive-compulsiveness and schizotypal dimensions by considering the mediating role of inferential confusion in a non-clinical sample.

\section{Materials and Methods}

\section{Study procedure and participants}

The study design was cross-sectional. The research population consisted of undergraduate students of Shahed University, Tehran in the academic year 2017-2018. There is no uniform strategy for determining sample size in Structural Equation Modeling (SEM) studies. As a rule of thumb, Loehlin (2004) considers a sample size of less than 100 to be inappropriate and more than 200 to be desirable. Kline (2011) also notes that the typical sample size in studies using SEM is 200. A total of 341 students (158 men and 183 women) participated in this study, which was adequate based on the minimum sample size. They were selected using the cluster sampling method as follows: 1) four faculties (out of eight faculties) were randomly selected; 2) According to the ratio of students in each faculty to the sample size, classes from each faculty were randomly selected and all students in those classes entered the study. Inclusion criteria were satisfaction with participation in the research and having no serious physical or psychological problems and exclusion criteria were dissatisfaction with participation in the research and having serious physical or psychological problems. The age of the subjects ranged from 18 to 29 years and their mean and standard deviation were 20.5 and 1.96 , respectively. Participants were explained about the general purpose of the research and after informed consent, they completed the following questionnaires. In addition, the subjects were free to refuse to continue completing the questionnaires and to leave the research. The questionnaires were presented in three different orders to control the effect of the order. After collecting data, they were analyzed using SEM and Bootstrapping method.

\section{Study measures}

\section{Obsessive-Compulsive Inventory-Revised (OCI-R)}

The OCI-R (Foa et al., 2002) has 18 items in six subscales: 1) washing, 2) hoarding, 3) checking/doubting, 4) ordering, 5) mental neutralizing, and 6) obsessing. Items are rated on a five-point Likert scale from 0 to 4 . Thus, the range of the total score is between 0 and 72 . Hajcak, Huppert, Simons, and Foa (2004) assessed the general population and showed good to excellent internal consistency (0.61-0.88) and test-retest reliability (0.54-0.77) for the total scale and subscales. The convergent and divergent validity of this scale was also confirmed. The reliability and validity of OCI-R in the Iranian sample were tested by Ghassemzadeh, Shams, Abedi, Karamghadiri, Ebrahimkhani, and Rajabloo (2011). The Cronbach's alpha and test-retest reliability for the total scale and subscales were ranged from 0.77 to 0.86 and 0.62 to 0.76 , respectively. Also, the convergent and divergent validity of the scale was confirmed by calculating its correlation coefficient with Maudsley Obsessional-Compulsive Inventory $(\mathrm{r}=0.57)$ and Beck Depression Inventory-II $(\mathrm{r}=0.39)$. In the present study, Cronbach's alpha for the total score of OCI-R was obtained 0.86 . 
Schizotypal Personality Questionnaire-Brief (SPQ-B)

The SPQ-B (Raine \& Benishay, 1995) has 22 items that the subject will answer yes or no to each of them. Each item will be given a score of zero or one; thus, the total scores fall within the range between 0 and 22. SPQ$\mathrm{B}$ measures schizotypal symptoms in three categories: disorganization (refer to disorganized schizotypy), interpersonal deficits (refer to negative schizotypy), and cog- nitive-perceptual dysfunction (refer to positive schizotypy). Cronbach's alpha coefficients $(0.72-0.80)$ and test-retest reliability coefficients $(0.86-0.95)$ for the total scale and subscales supported the reliability of this scale. The criterion validity of SPQ-B was confirmed by calculating correlational coefficients between the total score and its subscales with other clinical measures of schizotypal personality disorder $(0.34 \leq \mathrm{r} \leq 0.73, \mathrm{P}<0.05)$ (Raine

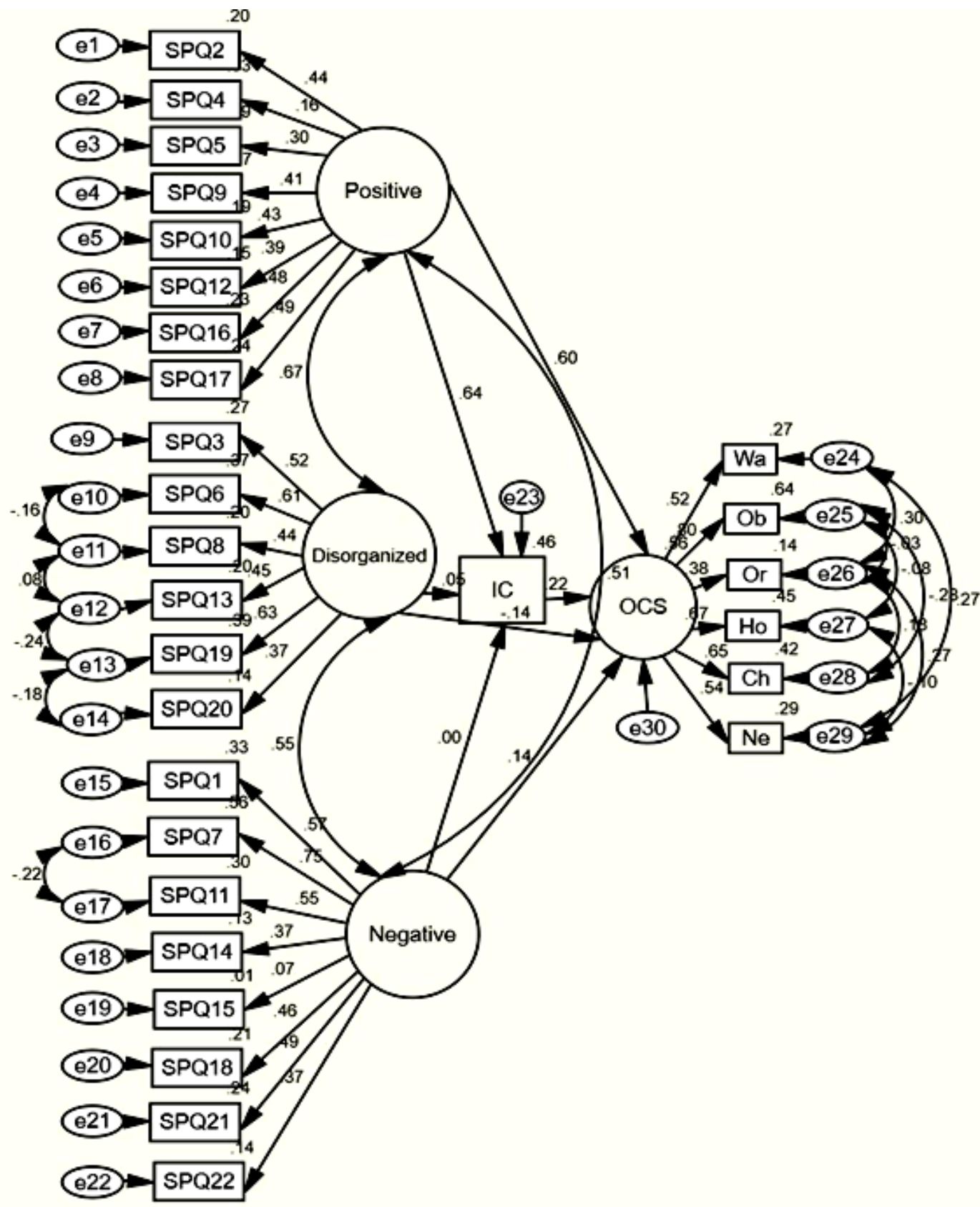

Figure 1. Fitted model with standard path coefficients

Positive: Positive Dimension of Schizotypy; Disorganized: Disorganized Dimension of Schizotypy; Negative: Negative Dimension of Schizotypy; IC: Inferential Confusion; OCS: Obsessive-Compulsive Symptoms; Wa: Washing; Ob: Obsessing; Or: Ordering; Ho: Hoarding; Ch: Checking; Ne: Neutralizing; SPQ1- SPQ22: SPQ-B questionnaire items. 
\& Benishay, 1995). The psychometric properties of the Persian version of the SPQ-B were demonstrated by Mohammadzadeh (2007). The test-retest reliability for the total scale and subscales was reported between 0.83 and 0.90. Also, Cronbach's alpha for total score and for subscales ranged from 0.66 to 0.83 . The convergent validity of this scale was confirmed by calculating its correlation coefficient with Schizotypal Trait questionnaire-A (STA; $\mathrm{r}=0.75, \mathrm{P}<0.01)$. In the present study, Cronbach's alpha for SPQ-B was obtained 0.76 .

\section{Inferential Confusion Questionnaire-Expanded Version (ICQ-EV)}

ICQ-EV (Aardema et al., 2010) is a one-dimensional questionnaire that has 30 items. Each item is rated on a Likert scale from strongly disagree (1) to strongly agree (6). The total score of ICQ-EV ranges from 30180. Cronbach's alpha in clinical (0.97) and nonclinical (0.96) samples showed very high internal consistency. Also, the results supported convergent, criterion, and clinical validity of ICQ-EV (Aardema et al., 2010). The reliability and validity of this scale in the Iranian sample were tested by Ghorbali, Shairi, and GholamiFesharaki (2018). Their results showed excellent internal consistency using Cronbach's alpha (0.95) and high testretest reliability $(\mathrm{r}=0.9)$ in a non-clinical sample. Also, convergent and divergent validity of the Persian version of ICQ-EV were confirmed. In the present study, Cronbach's alpha for ICQ-EV was obtained 0.93.

\section{Results}

The mean and standard deviation of the research variables were as follows: Positive schizotypy $=3.34 \pm 1.97$, Negative schizotypy $=3.7 \pm 2.07$, Disorganized schizo- typy $=1.94 \pm 1.63$, Inferential confusion $=92.92 \pm 23.21$, $\mathrm{OCS}=18.99 \pm 10.23$.

In order to investigate the mediating role of inferential confusion in the relationship between three dimensions of schizotypy and OCS, a proposed model was designed and examined by SEM. Before analyzing the data, assumptions of this analysis method, including univariate normality [by calculating skewness and kurtosis], multivariate normality [by calculating Mardia's coefficient (c.r.=4.69); critical value $\leq 5$ is indicative of data that are normally distributed (Bentler, 2005; as cited in Byrne, 2016)], and outlier data [by calculating Mahalanobis distance] were tested and approved. After applying some error correlations to the proposed model, the model was fitted with the data: $\chi^{2} / \mathrm{df}(1.54)$ was less than 3 , Goodness-of-Fit Index (GFI) (0.91) was more than 0.9, and RMSEA=0.04 (CI $(90 \%)=0.03-0.04)$ was less than 0.05 . The tested model and standard path coefficients are shown in Figure 1. Also, as shown in Table 1, among the three dimensions of schizotypy, only the direct effect of positive schizotypy on OCS $(\beta=0.60, \mathrm{P}<0.001)$ and inferential confusion $(\beta=0.64, \mathrm{P}<0.001)$ were statistically significant. Then, the Bootstrapping method was used to determine the significance of indirect effects from three dimensions of schizotypy to OCS. The results supported the mediating role of inferential confusion in the relationship between positive schizotypy and OCS $[\mathrm{CI}(95 \%)=0.01$ 0.25 ], but the mediating role of inferential confusion in the relationship between OCS and negative schizotypy $[\mathrm{CI}(95 \%)=-0.04-0.03]$, and disorganized schizotypy [CI $(95 \%)=-0.02-0.08]$ was not significant.

Table 1. Regression coefficients, standard errors, and significance level of the relationship between variables in the tested model

\begin{tabular}{llccc}
\hline \multicolumn{1}{c}{ Paths } & B & SE & B & P \\
\hline Positive schizotypy to obsessive-compulsive symptoms & 3.40 & 1.02 & 0.60 & $<0.001$ \\
\hline Positive schizotypy to inferential confusion & 67.07 & 14.37 & 0.64 & $<0.001$ \\
\hline Negative schizotypy to obsessive-compulsive symptoms & 0.60 & 0.35 & 0.14 & 0.09 \\
\hline Negative schizotypy to inferential confusion & 0.06 & 6.19 & 0.001 & 0.99 \\
\hline Disorganized schizotypy to obsessive-compulsive symptoms & -0.69 & 0.53 & -0.14 & 0.19 \\
\hline Disorganized schizotypy to inferential confusion & 4.58 & 9.30 & 0.05 & 0.62 \\
\hline Inferential confusion to obsessive-compulsive symptoms & 0.01 & 0.005 & 0.22 & 0.01 \\
\hline & & & PRACTICE In \\
\hline
\end{tabular}




\section{Discussion}

Consistent with the results obtained in the present study, it has been suggested that positive symptoms of schizotypy were associated with OCS more than negative and disorganized symptoms (Chmielewski \& Watson, 2008; Lee, Cougle, \& Telch, 2005; Shan et al., 2020; Suhr, et al., 2006; Zhang et al., 2019). Also, in line with these results, the presence of positive schizotypal symptoms in OCD patients can be associated with treatment failure (Mohammadzadeh et al., 2009; Moritz et al., 2004). These findings indicate the importance of paying attention to the heterogeneous dimensions of schizotypy in relation to the OCS (Poyurovsky, 2013). As predicted, our findings revealed that IC mediated the relationship between OCS and the positive dimension of schizotypy in a non-clinical sample. According to the obtained results, the direct and indirect (through IC) effects of the disorganized and negative dimension of schizotypy on OCS were not statistically significant.

Magical thinking is one of the components of positive schizotypy that has been considered in explaining the relationship between positive schizotypy and OCS. This component makes people vulnerable to experiencing OCS by increasing the likelihood of Thought-Action Fusion (TAF) (Lee et al., 2005). TAF is a kind of cognitive bias that has two subtypes: 1) likelihood TAF, in which a person believes that his/her thoughts can affect external realities as a cause, and 2) moral TAF, in which a person believes that thinking about morally forbidden thoughts is equivalent to doing it (Rachman, 1997). The likelihood and moral TAF make a person vulnerable to OCS by creating an extreme sense of responsibility for preventing harm and having pervasive and disturbing thoughts (Poyurovsky, 2013).

IC is another cognitive component that can partially explain the relationship between obsessive-compulsiveness and schizotypy. According to the IBA, OCD can be conceptualized as a disorder of the imagination; the person imagines a state of affairs and then considers it as a reality and acts accordingly (O'Connor, Aardema, \& Pelissier, 2005). Also, imagination has an important role in Delusional Disorders (DD), due to false beliefs that are far from reality (Aardema $\& \mathrm{Wu}, 2011$ ). Researchers consider schizotypy as a non-clinical form of delusional beliefs (Claridge et al., 1996). Therefore, imagination also plays a role in schizotypal phenomena. O'Leary (2015) considers obsessive-compulsiveness and schizotypy as unusual ways of thinking about the reality that are causally implausible and lie on a continuum of severity. Magical thinking and perceptual disturbances (which are considered as components of positive schizotypy( may reflect the pathological imaginative factor involved in the relationship between obsessive-compulsiveness and schizotypy. It seems that one of the possible reasons for explaining the relationship between positive schizotypy (but not negative and disorganized schizotypy) and OCS is related to the role of imagination.

Based on IBA, perceptual disturbances can be explained by using the concept of IC, which lies on a spectrum in terms of intensity. The more this feature intensifies in the thought process, the more one moves away from reality. Therefore, he/she is more likely to experience perceptual disturbances (Ardema, Emmelkamp, \& Connor 2005; Aardema, Kleijer, Trihey, O’Connor, \& Emmelkamp 2006). Thus, this approach considers OCD and DD as belief disorders and introduces IC as one of the important common components between them (O'Leary, 2015). The insignificance of the direct effects of negative and disorganized dimensions of schizotypy to OCS may be an emphasis on the fact that OCD is a belief disorder and the overlap between schizotypy and obsessive-compulsiveness can be better explained by a cognitive factor, such as IC. This finding could have important implications in the treatment of patients with symptoms of the two disorders. As mentioned, the comorbidity between OCS and schizotypal personality traits is very important. Research evidence has shown that OCD patients with positive symptoms of schizotypy (compared to negative and disorganized symptoms) benefit less from standard behavioral and psychopharmacological treatments, have a greater treatment resistance, and generally have a poor prognosis. In this regard, the results of the present study as a preliminary step showed that the construct of "inferential confusion" is able to partially explain the relationship between OCS and positive schizotypy. Therefore, examining the relationships between schizotypal personality traits and OCS based on Inference-Based Therapy (IBT) can increase the knowledge of specialists and experts about this group of patients and the effectiveness of IBT is promising for this group of patients.

It should be noted that this research has some limitations that need to be considered. First, due to the crosssectional nature of the research design, it is not possible to infer causal relationships between variables. Second, the sample studied in this study was a non-clinical sample and its participants were students of a single university, which limits the generalizability of the research results to the clinical and general populations. Third, the data in this study were collected using self-report questionnaires. Another limitation of this study was that the subtypes of OCD were not studied separately. It is suggested that in 
future studies, the model of this study be examined in broader samples, especially in clinical samples. Also, future studies can use methods other than self-report questionnaires to collect data, when possible. In addition, by considering the subtypes of OCD, a more accurate understanding of the relationship between OCS and schizotypal traits can be obtained and the role of IC in explaining the relationship between these two disorders can be better understood. Also, it is suggested that using a treatment protocol based on inferential confusion (IC) in patients with symptoms of both OCD and SPD can provide more evidence to clarify the role of the IC in these two disorders and this protocol can be examined to show how this reasoning process relates to the treatment outcome.

\section{Conclusion}

The findings of this study provided evidence that emphasized the heterogeneous nature of the schizotypy in the relationship with OCS. Also, the results showed that IC can be considered as one of the common components in OCS and positive schizotypy.

\section{Ethical Considerations}

\section{Compliance with ethical guidelines}

Participants were explained about the general purpose of the research and confidentiality of the information and after informed consent, they completed the questionnaires. In addition, the subjects were free to refuse to continue completing the questionnaires at any time.

\section{Funding}

This research did not receive any grant from funding agencies in the public, commercial, or non-profit sectors.

\section{Conflict of interest}

There are no conflicts of interests.

\section{References}

Aardema, F., Emmelkamp, P. M. G., \& O'Connor, K. P. (2005). Inferential confusion, cognitive change and treatment outcome in obsessive-compulsive disorder. Clinical Psycholoy and Psychotherapy, 12(5), 337-45. [DOI:10.1002/cpp.464]

Aardema, F., \& Wu, K. D. (2011). Imaginative, dissociative, and schizotypal processes in obsessive-compulsive symptoms. Journal of Clinical Psychology, 67(1), 74-81. [DOI:10.1002/ jclp.20729] [PMID]
Aardema, F., Kleijer, T. M., Trihey, M., O'Connor, K., \& Emmelkamp, P. M. (2006). Processes of inference, schizotypal thinking, and obsessive-compulsive behaviour in a normal sample. Psychological Reports, 99(1), 213-20. [DOI:10.2466/ PR0.99.5.213-220] [PMID]

Aardema, F., Wu, K. D., Careau, Y., O'Connor, K., Julien, D., \& Dennie, S. (2010). The expanded version of the inferential confusion questionnaire: Further development and validation in clinical and non-clinical samples. Journal of Psychopathology and Behavioural Assessment, 32(3), 448-62. [DOI:10.1007/ s10862-009-9157-x]

Byrne, B. M. (2016). Structural equation modeling with Amos: Basic concepts, applications, and programming. New York: Routledge. [DOI:10.4324/9781315757421]

Chmielewski, M., \& Watson, D. (2008). The heterogeneous structure of schizotypal personality disorder: Item-level factors of the schizotypal personality questionnaire and their associations with obsessive-compulsive disorder symptoms, dissociative tendencies, and normal personality. Journal of Abnormal Psychology, 117(2), 364-76. [DOI:10.1037/0021-843X.117.2.364]

Claridge, G., McCreery, C., Mason, O., Bentall, R., Boyle, G., \& Slade, P., et al. (1996). The factor structure of 'schizotyp$\mathrm{al}^{\prime}$ traits: A large replication study. British Journal of Clinical Psychology, 35(1), 103-15. [DOI:10.1111/j.2044-8260.1996. tb01166.x] [PMID]

Foa, E. B., Huppert, J. D., Leiberg, S., Hajcak, G., Langner, R., \& Kichic, R., et al. (2002). The obsessive-compulsive inventory: Development and validation of a short version. Psychological Assessment, 14(4), 485-96. [DOI:10.1037/1040-3590.14.4.485] [PMID]

Ghassemzadeh, H., Shams, G., Abedi, J., Karamghadiri, N., Ebrahimkhani, N., \& Rajabloo, M. (2011). Psychometric properties of a Persian-language version of the Obsessive-Compulsive Inventory Revised (OCI-R-Persian). Psychology, 2(3), 210-5. [DOI:10.4236/psych.2011.23032]

Ghorbali, A., Shairi, M., \& Gholami-Fesharaki, M. (2018). [Preliminary study of psychometric properties of the persian version of the expanded version of the Inferential Confusion Questionnaire (Icq-Ev) in a non-clinical sample (Persian)] Journal of Psychological Science, 17(67), 373-9. http:// psychologicalscience.ir/article-1-131-fa.html

Hajcak, G., Huppert, J. D., Simons, R. F., \& Foa, E. B. (2004) Psychometric properties of the OCI-R in a college sample. Behavior Re-search \& Therapy, 42(1), 115-23. [DOI:10.1016/j. brat.2003.08.002]

Kline, R. B. (2011). Principles and practice of structural equation modeling. New York: Guilford Press. https://www.google. com/books/edition/Principles_and_Practice_of_

Lee, H. J., Cougle, J. R., \& Telch, M. J. (2005). Thought-action fusion and its relationship to schizotypy and OCD symptoms. Behaviour Research and Therapy, 43(1), 29-41. [DOI:10.1016/j. brat.2003.11.002] [PMID]

Loehlin, J. C. (2004). Latent variable models: An introduction to factor, path, and structural equation analysis. London: Lawrence Erlbaum Associates Publishers. [DOI:10.4324/9781410609823]

Mohammadzadeh, A. (2007). The study of psychometric properties of Schizotypal Personality Questionnaire-Brief (SPQ-B) International Journal of Psychology, 1(2), 47-61. http://ijpb.ir/ article-1-109-fa.html 
Mohammadzadeh, A. (2010). Relationship between the threefactor schizotypal personality model and obsession: An investigation among a non-clinical sample (Persian)]. Contemporary Psychology, 5(1), $42-50 \mathrm{http}: / /$ bjcp.ir/browse php?a_id=273\&sid=1\&slc_lang=en

Mohammadzadeh, A., Borjali, A., Sohrabi, F., \& Delavar, A. (2009). [The effect of co-morbidity of positive and negative schizotypal personality traits on treatment outcome of obsessive-compulsive subtypes (Persian)]. Journal of Behavioral Science Research, 7(1), 13-24. [DOI:20.1001.1.17352029.1388.7.1.2.5]

Moritz, S., Fricke, S., Jacobsen, D., Kloss, M., Wein, C., \& Rufer, M., et al. (2004). Positive schizotypal symptoms predict treatment outcome in obsessive-compulsive disorder. Behavior Research and Therapy, 42(2), 217-27. [DOI:10.1016/S00057967(03)00120-7] [PMID]

Norman, R. M., Davies, F., Malla, A. K., Cortese, L., \& Nicholson, I. R. (1996). Relationship of obsessive-compulsive symptomatology to anxiety, depression and schizotypy in a clinical population. British Journal of Clinical Psychology, 35(4), 553-66. [DOI:10.1111/j.2044-8260.1996.tb01211.x] [PMID]

O'Connor, K. P., \& Robillard, S. (1995). Inference processes in obsessive-compulsive disorder: Some clinical observations. Behavior Research and Therapy, 33(8), 887-96. [DOI:10.1016/00057967(95)00042-V]

O'Connor, K., Aardema, F., \& Pelissier, M. C. (2005). Beyond reasonable doubt: Reasoning processes in obsessive-compulsive disorder and related disorders. Chichester: John Wiley \& Sons. [DOI:10.1002/0470030275]

O'Leary, N. (2015). The relationship between inferential confusion, obsessive compulsiveness, schizotypy and dissociation in a non-clinical sample [PhD dissertation]. Guildford: University of Surrey. https://openresearch.surrey.ac.uk/ esploro/outputs/doctoral/The-relationship-between-infer

Paradisis, S. M., Aardema, F., \& Wu, K. D. (2015). Schizotypal, dissociative, and imaginative processes in a clinical OCD sample. Journal of Clinical Psychology, 71(6), 606-24. [DOI:10.1002/ jclp.22173] [PMID]

Perris, F., Fabrazzo, M., De Santis, V., Luciano, M., Sampogna, G., \& Fiorillo, A., et al. (2019). Comorbidity of obsessive-compulsive disorder and schizotypal personality disorder: Clinical response and treatment resistance to pharmacotherapy in a 3-year follow-up naturalistic study. Front Psychiatry, 10, 386. [DOI:10.3389/fpsyt.2019.00386] [PMID] [PMCID]

Poyurovsky, M. (2013). Schizo-obsessive disorder. Cambridge: Cambridge University Press. [DOI:10.1017/ CBO9780511686931]

Rachman, S. (1997). A cognitive theory of obsessions. Behavior Research and Therapy, 35(9), 793-802. [DOI:10.1016/S00057967(97)00040-5]

Raine, A., \& Benishay, D. (1995). The SPQ-B: A brief screening instrument for schizotypal personality disorder. Journal of Personality Disorders, 9(4), 346-55. [DOI:10.1521/ pedi.1995.9.4.346]

Raynal, P., Goutaudier, N., Nidetch, V., \& Chabrol, H. (2016). Typology of schizotypy in non-clinical young adults: Psychopathological and personality disorder traits correlates. Psychiatry Research, 246, 182-7. [DOI:10.1016/j.psychres.2016.09.042] [PMID]
Rossi, A., \& Daneluzzo, E. (2002). Schizotypal dimensions in normal and schizophrenic patients: A comparison with other clinical samples. Schizophrenia Research, 54(1), 67-75. [DOI:10.1016/S0920-9964(01)00353-X]

Shan, H., Zhang, R., Jiang, S., Wang, Y., Liu, Y., \& Cheung, E. F. C., et al. (2020). Schizotypal and obsessive-compulsive traits: Co-occurrence rate and relationship with executive function, emotion experience, and emotion expressivity in college students. PsyCh Journal, 9(5), 749-59. [DOI:10.1002/pchj.372]

Sobin, C., Blundell, M. L., Weiller, F., Gavigan, C., Haiman, C., \& Karayiorgou, M. (2000). Evidence of a schizotypy subtype in OCD. Journal of Psychiatry Research, 34(1), 15-24. [DOI:10.1016/ S0022-3956(99)00023-0] [PMID]

Suhr, J. A., Spitznagel, M. B., \& Gunstad, J. (2006). An obsessivecompulsive subtype of schizotypy: Evidence from a nonclinical sample. The Journal of Nervous and Mental Disease, 194(11), 884-6. [DOI:10.1097/01.nmd.0000243929.45895.d9] [PMID]

Tallis, F., \& Shafran, R. (1997). Schizotypal personality and obsessive compulsive disorder. Clinical Psychology and Psychotherapy, 4(3), 172-8. [DOI:10.1002/(SICI)10990879(199709)4:33.0.CO;2-\#]

Weintraub, M. J., Brown, C. A., \& Timpano, K. R. (2018). The relationship between schizotypal traits and hoarding symptoms: An examination of symptom specificity and the role of perceived cognitive failures. Journal of Affective Disorders, 237, 10-7. [DOI:10.1016/j.jad.2018.04.121] [PMID]

Zhang, R. T., Zhou, H. Y., Wang, Y. M., Yang, Z. Y., Wang, Y., \& So, S. H., et al. (2019). Network analysis of schizotypal personality traits and their association with other subclinical psychiatric features. Asian Journal of Psychiatry, 44, 209-16. [DOI:10.1016/j.ajp.2019.08.005] [PMID] 